\title{
Seroconversion and adverse reaction of live-attenuated (OKA strain) varicella vaccine
}

\author{
Yulchair Ramli, MD; Sri Rezeki HA, MD, PhD; Arwin P Akib, MD
}

\begin{abstract}
Background Live-attenuated varicella vaccine was used to prevent Shingle disease.

Objective The aim of this study was to find out seroconversion rate and safety of this vaccine.

Methods An open clinical trial was conducted on 313 children at Cipto Mangunkusumo Hospital, Jakarta between March and October 1998. The data were analyzed to distribution frequency and tables.

Results Only 43 out of 356 (12.1\%) children had positive antivaricella antibody before vaccination. Seroconversion examination by indirect immunoflouresence method was done only on randomly 150 of $313(50 \%)$ children due to financial limitation. It was found that $99.3 \%$ of patients had positive seroconversion. Among 298 children who were followed, the nature of symptom of adverse reaction was mostly general than local. Only one patient had local symptom after vaccination that appeared within 7 days of the follow up period.

Conclusion Varicella vaccine (OKA strain) gives high seroconversion rate with minimal adverse events [Paediatr Indones 2003;43:171-176].
\end{abstract}

Keywords: varicella vaccine, seroconversion, adverse reaction.

$\mathrm{V}$ aricella is a highly contagious infectious disease. After an 11-20 day incubation period, an easily recognized clinical manifestation develops, consisting of papulo-vesicular rash which progresses into noninfectious dry crust after 5-6 days. ${ }^{1}$

The incidence of the disease is influenced by geographic factor. In temperate climate region more than $90 \%$ of adults with a history of previous varicella infection are serologically positive against varicella-zooster virus (VZV), whereas in adults without history of previous infection, it is 70\%, suggesting a previous subclinical varicella infection. ${ }^{2,3}$ The highest incidence of varicella infection in children is at the age of 4-9 years. ${ }^{1}$ Meanwhile in the tropical region, the highest incidence is in young adults of 15 24 year age group. ${ }^{3-5}$ Serological study in Indonesia found a high seronegativity ( $>50 \%$ ) among the age beyond 15 year-old. ${ }^{6}$ The disease is preventable by the administration of an attenuated varicella virus vaccine developed initially by Takahashi on 1974 and licensed in US in 1995.7,8 This vaccine had been tested in more than 2000 healthy children and adolescents in Japan in 1988 and proven to be immunogenic and safe. Seroconversion among one year age and two year age children were $100 \%$ and $98 \%$ respectively. Skin rash as an adverse reaction developed in six percents of subjects, with an average of 5 skin lesions. ${ }^{8}$ This vaccine has been available in the market in Indonesia since 1997. This study aimed to assess the proportion of negative seroconversion and adverse events following administration of a live-attenuated varicella vaccine (OKA strain) in healthy children aged $1-12$ years in Jakarta.

From the Department of Child Health, Medical School, University of Indonesia, Jakarta.

Reprint requests to: Yulchair Ramli, MD, Department of Child Health, Medical School, University of Indonesia, Cipto Mangunkusumo Hospital, Jakarta, Indonesia. Tel. 62-21-3907740, Fax. 62-21-3907743. 


\section{Methods}

This study was an open, non-comparative clinical trial on three age groups of children to find out the seroconversion and adverse events following administration of a live-attenuated varicella vaccine. The study was conducted in the Department of Child Health, Medical School, University of Indonesia/Cipto Mangunkusumo Hospital (FKUI-RSCM), Jakarta from May 1998 until October 1998. Subjects were healthy children aged 12 months-12 years whose parents agreed to participate. Inclusion criteria were healthy children aged 12 months-to 12 years, had negative antivaricella antibody, were not in any immunosupressant or oral antituberculosis medication, and their parents signed the informed consent. Two weeks prior to vaccination, each child was examined for antivaricella antibody titer. One hundred and fifty children were reexamined for post vaccination antivaricella antibody to assess the seroconversion.

The vaccine was injected after the subjects underwent physical examination and were considered health. The parents were instructed to measure the body temperature and local or general clinical manifestations (including papules or vesicles) following the injection and to write down on a certain diary card. Local manifestations were assessed based on: 1) local adverse reactions (muscleache, size of redness and size of swelling); 2) score of reaction: $0=$ none, $1=$ less than $5 \mathrm{~mm}, 2=5-20 \mathrm{~mm}, 3=$ more than $20 \mathrm{~mm}$. General manifestations were assessed based on body temperature intensity and number of skin papules or vesicles. The relationships of both local and general reaction with the vaccination were categorized into: PB (probable: probable direct relation between adverse effect and vaccination), SU (suspected/ reasonable probability: no direct relation was proven but there was other reasonable probability), UL (unlikely: any other causes besides the vaccine), NR (non related: adverse effect unrelated to the vaccine). Body temperature intensity was divided into grade 1 (mild $=37.5^{\circ}-39^{\circ} \mathrm{C}$ ) and grade $2\left(\right.$ severe $\left.\geq 39^{\circ} \mathrm{C}\right)$. The parents could bring the child any time to be examined by the authors if necessary. Assessment of local manifestation was done during the week after vaccination; meanwhile the general manifestation, fever and rashes, were assessed for 42-day period as well.
Samples of pre and post vaccination serum were stored at $-20^{\circ} \mathrm{C}$ before assessment. Prevaccination serums were assessed at the Prodia Bioanalytical Research Corporation (BARC) laboratory, Jakarta, using ELISA method (Enzygnost anti VZV IgG). Antivaricella antibody titer $>10 \mathrm{mU} / \mathrm{mL}$ was considered positive level. Seroconversion examination was done only on randomly selected 150 blood samples due to financial limitation. They were examined at the Smith Kline Beecham Biological Laboratory in Brussels, Belgium for reassessment of prevaccination and post vaccination antivaricella antibody titer, using the Indirect Immunofluorescence (IIF) method, and considered positive if IIF titer was $>4 \mathrm{mU} / \mathrm{mL}$. This study was approved by the Ethical Committee of University of Indonesia.

Data were processed using SPSS program and presented in tables, graphs, and text.

\section{Results}

Within the study period 356 children underwent antivaricella antibody serological examination before vaccination, consisting of 189 boys (53.1\%) and 167 girls (46.9\%); 1-3 year age group consisted of 113 children $(31.7 \%) ;>3-7$ year age, 136 children (38.2\%); and >7-12 year age, 107 (30.1\%). Positive antivaricella antibodies were found in 43 children (12.1\%); 18 boys (5.1\%), 25 girls (7.0\%) and 2 children of $1-3$ year age group $(0.6 \%), 16$ children of $>3-7$ year age group $(4.5 \%)$ and 25 children of $>7$. 12 year age group (7.0\%).

The older the age group, the lower the seronegative proportion was. Figure 1 shows the distribution of seropositive children according to age and sex. Male to female ratio of seropositive children were 1:1.39. Seropositive children were most frequently found in the 7-12 year age group and the youngest was 24 month- old. No children less than one yearold was seropositive.

\section{Seroconversion}

One hundred and fifty children had undergone serological examination by indirect immunofluorescence method to assess the seroconversion of the antivaricella antibody. One subject was excluded due 
Yulchair Ramli et al: Seroconversion and adverse reaction of varicella vaccine

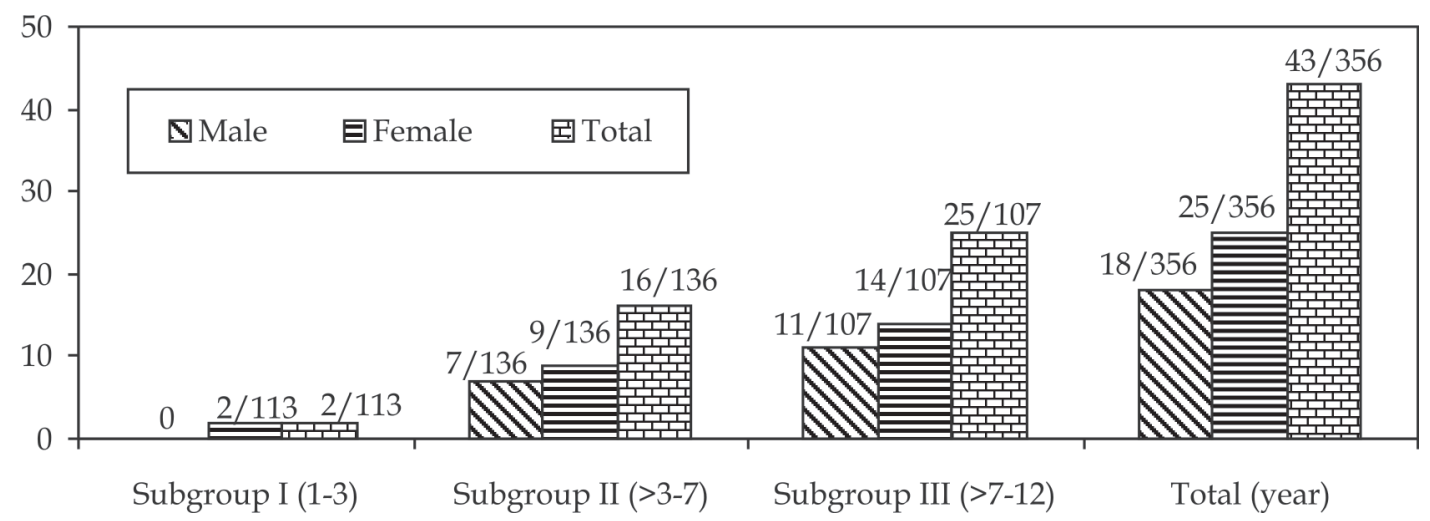

Figure 1. Distribution of positive antivaricella antibody by sex and age.

to missing laboratory result. Two subjects were excluded due to seropositive antivaricella antibody by IIF method. Seroconversion distribution result based on age showed that all of 47 children in the 1-3 year age group and all of 48 children in the $>7-12$ year age group had seropositive antivaricella antibody. Only 1 child out of 52 children in the $>3-7$ year age group still had negative titer in IIF examination.

Forty-two days after vaccination, 146 out of 147 subjects $(99.3 \%)$ had seropositive antivaricella antibody by IIF examination.

\section{Post varicella vaccination adverse events}

During the study period, 298 out of 313 subjects were assessed for adverse events of varicella vaccination. Table 1 shows their characteristics.

General and local manifestations of adverse events following the vaccination are shown in Table 2 and 3.
General manifestations (fever or rashes) were more frequently found than local manifestations $(8.7 \%$ vs. $0.3 \%)$. The clinical manifestations were most frequently found in the age group of $>3-7$ years.

\section{Local symptoms and signs}

During the seven day observation after vaccination only 1 out of 297 subjects $(0.3 \%)$ in the $>3-7$ year age group had local manifestation, suffered from the first grade of pain that resolved within 2 days.

\section{General symptoms and signs}

Observation on day 7 and 42 post-vaccination found that $g$ eneral manifestation was only fever.

Out of 293 children who were followed until the end of the study period, 24 subjects had fever. From 24 subjects with fever, 10 children of the 1-3 year age group, 7 children of the $>3-7$ year age group and 4 of the $>7-12$ year age group had mild fever. Three children of the $>3-7$ year age group had severe fever. Ten

Table 1. Demographic characteristics of subjects

\begin{tabular}{llrcrcc}
\hline \multicolumn{2}{l}{ Sub-group } & Sex & $\begin{array}{c}\text { Mean of age } \\
\text { (months) }\end{array}$ & SD & Minimum & Maximum \\
\hline I & Female & 47 & 23.3 & 7.2 & 10 & 35 \\
& Male & 57 & 23.1 & 7.2 & 9 & 36 \\
& Total & 104 & 22.6 & 7.2 & 9 & 36 \\
II & Female & 58 & 57.7 & 15.8 & 37 & 84 \\
& Male & 56 & 59.6 & 14.8 & 37 & 83 \\
& Total & 114 & 58.6 & 15.3 & 37 & 84 \\
III & Female & 38 & 109.2 & 17.6 & 86 & 155 \\
& Male & 42 & 107.5 & 20.4 & 85 & 154 \\
& Total & 80 & 108.3 & 19.0 & 85 & 155 \\
\hline \multirow{2}{*}{ Total } & Female & 143 & 60.7 & 36.0 & 10 & 155 \\
& Male & 155 & 58.8 & 36.8 & 9 & 154 \\
& Total & 298 & 59.4 & 36.4 & 9 & 155 \\
\hline
\end{tabular}


Paediatrica Indonesiana

Table 2. General and local manifestations by SeX

\begin{tabular}{lclllll}
\hline Sex & \multicolumn{3}{c}{ General manifestation } & \multicolumn{3}{c}{ Local manifestation } \\
& Yes) & No & Total & Yes & No & Total \\
\hline Male & 13 & 148 & 161 & 1 & 160 & 161 \\
& $(4.4)$ & $(49.6)$ & $(54.0)$ & $(0.3)$ & $(53.9)$ & $(54.2)$ \\
Female & 13 & 124 & 137 & 0 & 136 & 136 \\
& $(4.4)$ & $(41.6)$ & $(46.0)$ & $(0.0)$ & $(45.8)$ & $(45.8)$ \\
\hline Total & 26 & 272 & 298 & 1 & 296 & 297 \\
& $(8.7)$ & $(91.3)$ & $(100)$ & $(0.3)$ & $(99.7)$ & $(100)$ \\
\hline
\end{tabular}

Table 3. General and local manifestations by age group

\begin{tabular}{lclllll}
\hline Subgroup & \multicolumn{3}{c}{ General manifestation } & \multicolumn{2}{c}{ Local manifestation } \\
& \multicolumn{3}{c}{$(\%)$} & & & $\begin{array}{l}\text { (\%) } \\
\end{array}$ \\
& Yes & No & Total & Yes & No & Total \\
\hline I & 10 & 95 & 105 & 0 & 105 & 105 \\
& $(3.4)$ & $(31.9)$ & $(35.2)$ & $(0.0)$ & $(35.3)$ & $(35.3)$ \\
II & 12 & 103 & 115 & 1 & 114 & 115 \\
& $(4.0)$ & $(34.6)$ & $(38.6)$ & $(0.3)$ & $(38.4)$ & $(38.7)$ \\
III & 4 & 74 & 78 & 0 & 77 & 77 \\
& $(1.3)$ & $(24.8)$ & $(26.2)$ & $(0.0)$ & $(25.8)$ & $(25.9)$ \\
\hline Total & 26 & 272 & 298 & 1 & 296 & 297 \\
& $(8.7)$ & $(91.3)$ & $(100)$ & $(0.3)$ & $(99.7)$ & $(100)$ \\
\hline
\end{tabular}

children of 24 had fever which was related to the vaccination. Eight children had mild fever related to vaccination (4 in the subgroup I, 2 in subgroup II, and 2 in subgroup III). In two children of the 3-7 year age group, severe fever noted was related to vaccination.

Observation for skin rash on day 7 to 42 found that 4 children out of 293 had it. One child of the 1 3 year age group and 3 children of the $>3-7$ year age group had mild skin rash. Two of them had abortive vesiculous rash 6 days after vaccination.

\section{Discussion}

\section{Age and sex}

Seronegative antivaricella antibody was found in 313 out of 356 subjects (prevalence rate was $87.9 \%$ ), mostly occurred in males $(90.5 \%$ vs. $85 \%)$. Studies both in temperate climate countries and tropical countries found similar result that sex was not a determinant factor for seronegativity. ${ }^{3-6,10,11}$

The prevalence of seropositive antivaricella antibody was $12.0 \%$, increasing with age among the age groups. This result was lower than that of the study in Yogyakarta in 1998 which was 30\%, 44\%, 58\% in the age group of 4, 5-9, and 10-14 year, respectively. ${ }^{6}$ Our result was also lower than that of other tropical countries. ${ }^{3-4,10,11}$ A study by Ooi in Singapore found $4 \%$ in the $0-4$ year of age, $21 \%$ in $5-14$ year of age, and $41 \%$ in 15-24 year of age. ${ }^{4}$ Other study in Itahari, Nepal found $25 \%, 64.7 \%, 81.8 \%$ among the $1-4,5-9$, and $10-14$ year age repectively. 10 These data show that the majority of children less than 14 year age are still susceptible to varicella. The data supports the suggestion that virus transmission is lower in the tropical countries than in the temperate climate countries, due to the faster transmission of virus in the temperate climate countries. ${ }^{12}$ A study in Bengali, West India found that 96.6\% (148/153) subjects from densely populated city were positive for antivaricella antibody, compared to $69.9 \%(172 / 246)$ subjects from rural area. ${ }^{12}$ Population density factor was said to increase the transmission of VZ virus. This condition leads to a low natural immunity level to $\mathrm{VZ}$ virus in children less than 12 year-old so that infection is more frequently found in older children. ${ }^{12}$

\section{Seroconversion of varicella antibody}

This study found $99.3 \%$ positive seroconversion distributed among the age groups. Previous studies found high positive seroconversion of varicella vaccine 
among children of all age groups. ${ }^{7,12-15}$ White found 96\% (2381/2475), using gp ELISA method. ${ }^{17}$ The highest seroconversion was found among the 12-15 year age group (98\%), and the lowest was among the 13-17 year-old. Other study found $98.6 \%$ in children of $9-36$ month-old. ${ }^{18}$

One study done in Japan found positive seroconversion in $93.6 \%$ among 973 children. ${ }^{19}$ The proportion was decreasing with the increase of age, from $96.0 \%$ among the one year-age to $75 \%$ at the age beyond 11 years. Age influences the seroconversion level and antibody titer. This study did not found similar condition probably due to the small number of subjects compared to the Japan's study.

\section{Post varicella vaccination adverse events}

The varicella vaccine seemed to be well tolerated without any serious adverse reactions during the observation period. ${ }^{15-20}$ During the 42 day observation, $8.7 \%$ of subjects had fever and skin rash as the general manifestations, $0.3 \%$ had pain at the injection site as the local manifestation. This result was similar to that of the study done in Japan which found $5.4 \%$ of subjects with redness at the injection site on day 0-2 post vaccination and $0.1 \%$ on day $7-20$ post vaccination, ${ }^{19}$ whereas other study found $13 \%$ of 3303 healthy children and adolescents with pain at the injection site as well. ${ }^{17}$

This study showed that during the observation on day $7-42$ post vaccination, $8.2 \%$ of subjects had fever beyond $37.5^{\circ} \mathrm{C}$, three of them had fever beyond $39^{\circ} \mathrm{C}$. The Japan's study ${ }^{19}$ found $10.6 \%$ had fever beyond $37.5^{\circ} \mathrm{C}$, while other study ${ }^{17}$ found $15 \%$.

This study found that 10 out of 24 subjects had fever related to the vaccination, whereas the rest had it from a respiratory tract infection, which occurred during the observation period. The result seemed to be similar with White's study. ${ }^{17}$ Abortive vesicles in our study were found in $1.36 \%$ of subjects (4/293), compared to the Japan's study who found $4.8 \%(45 / 943) .{ }^{19}$

This study concluded that the majority of Indonesian children less than 12 year-age do not have antivaricella antibody. Varicella vaccine (OKA strain) has given high seroconversion rate with minimal adverse events.

\section{References}

1. Preblud SR, Orenstein WA, Bart KJ. Varicella: clinical manifestation, epidemiology and health impact in children. Pediatr Inf Dis 1984;3:505-9.

2. Taylor-Wiedeman J, Yamashita K, Miyamura K, Yamazaki S. Varicella-zoster virus prevalence in Japan: no significant change in decade. Jpn J Med Sci Bio 1989;42:1-11.

3. Venkitaraman AR, Seigneurin JM, Lenoir GM, John TJ. Infection due to the human herpes viruses in southern India: a seroepidemiological survey. Int J Epidemiol 1986;15:561-6.

4. Ooi PL, Goh KT, Dorasingham S, Ling AE. Prevalence of varicella-zoster virus infection in Singapore. Southeast Asean J Trop Med Pub Health 1992;23:22-5.

5. Alvarez y Munoz MT, Torres J, Damasio-Santana L, Gomez A, Fernandez-Quintanilla G, Tapia-Conyer R, et al. Susceptibility to varicella-zoster infection in individual 1 to 29 years of age in Mexico. Arch Med Res 1999;30:60-3.

6. Juffrie M, Graham RR, Tan RI, Widjaja S, Muhjadi S, Well J, et al. Seroprevalence of hepatitis A virus and varicella zoster antibodies in a Javanese community (Yogyakarta, Indonesia). Southeast Asean J Trop Med Public Health 2000;31:21-4

7. Chartrand SA. Varicella vaccine. Pediatr Clin North Am 2000;47:1-16.

8. Takahashi M. The varicella vaccine. Vaccine development. Infect Dis Clin North Am 1996;10:1-12.

9. Zoulek G. Laboratory markers of immunity to varicella-zoster virus. Postgrad Med J 1985;61 Suppl 4:47-52.

10. Kubo T, Rai SK, Nakanishi M, Yamano T. Seroepidemiological study of herpes viruses in Nepal. Southeast Asean J Trop Med Public Health 1991;22:323-5.

11. Zhou T, Wang J, Qiu P. Investigation on sera prevalence rate of varicella and immunogenicity of varicella vaccine in healthy children. Chinese J Epidemiol 1998;19:271-3.

12. Mandal BK, Mukherjee PP, Murphy C, Mukherjee R, Naik T. Adult susceptibility to varicella in the tropics is a rural phenomenon due to the lack of previous exposure. J Infect Dis 1998;178 Suppl 1:S52-4.

13. Takahashi M. Clinical overview of varicella vaccine: development and early studies. Pediatrics 1986;78 Suppl 1:736-41. 


\section{Paediatrica Indonesiana}

14. Takahashi M, Kamiya H, Baba K. Clinical experience with Oka live varicella vaccine in Japan. Postgrad Med J 1985;61:61.

15. Gershon AA. Viral vaccines of the future. Pediatr Clin North Am 1990;37:689-707.

16. Andre FE. Worldwide experience with the OKA-strain live varicella vaccine. Postgrad Med J 1985;61 Suppl 4:113-20.

17. White CJ, Kuter BJ, Hildebrand CS, Isganitis KL, Matthews H, Miller WJ, et al. Varicella vaccine (VARIVAX) in healthy children and adolescents: result from clinical trials, 1987 to 1989. Pediatrics 1991;87:604-10.
18. Meurice F, De Bouver J, Vandevoorde D, Woods S, Bogaerts $\mathrm{H}$. Immunogenicity and safety of a live attenuated varicella vaccine (OKA/SB Bio) in healthy children. J Infect Dis 1996;174 Suppl 3:S324-9.

19. Ozaki T, Nishimura N, Kajita Y. Experience with live attenuated varicella vaccine (OKA strain) in healthy Japanese subjetcs; 10-year survey at pediatric clinic. Vaccine 2000;18:2375-80.

20. Watson BM, Laufer DS, Kuter BJ, Staehle B, White CJ. Safety and immunogenicity of a combined live attenuated measles, mumps, rubella, and varicella vaccine (MMR II V) in healthy children. J Infect Dis 1996;173:731-4. 